\title{
Synthesis, structural elucidation and biological activities of some novel sulfonyl hydrazones as antibacterial agents
}

\author{
Sevil ŞENKARDEŞ 1 * (D), Merve Eylül KIYMACI ${ }^{2}$ (D), Kübra KALE 3 (D), İsa Murat KOZANOĞLU 3 (D), \\ Banu KAŞKATEPE 4 (D), Ş. Güniz KÜÇÜKGÜZEL ${ }^{1}$ \\ 1 Department of Pharmaceutical Chemistry, Faculty of Pharmacy, Marmara University, Başıbüyük 34854 İstanbul, \\ Turkey. \\ 2 Department of Pharmaceutical Microbiology, Gulhane Faculty of Pharmacy, University of Health Sciences, Etlik- \\ Keçiören 06018 Ankara, Turkey \\ 3 Faculty of Pharmacy, Marmara University, Başıüyük 34854 İstanbul, Turkey. \\ 4 Department of Pharmaceutical Microbiology, Faculty of Pharmacy, Ankara University, Yenimahalle 06560 Ankara, \\ Turkey \\ * Corresponding Author. E-mail: sevil.aydin@marmara.edu.tr (S.Ş.); Tel. +90-216-777 5200.
}

Received: 23 December 2020 / Revised: 11 February 2021/ Accepted: 15 February 2021

ABSTRACT: In this study, some $N^{\prime}$-(substituted arylmethylidene)-4-nitrobenzenesulfonohydrazide derivatives were synthesis by reacting various aldehydes and 4-nitrobenzenesulfonohydrazide. The structural characterization of the compounds was performed by IR, ${ }^{1} \mathrm{H}-\mathrm{NMR}$ and TOF-MS (compounds $\mathbf{3 b}$ and $\mathbf{3 e}$ ) spectroscopic data besides elementel analyses results. The antibacterial activities of these compounds were examined against some bacteria species. The compounds showed the highest activity against Pseudomonas aeruginosa ATCC 27853. Also, anti-quorum sensing activities have been determined using a biosensor bioassay with Chromobacterium violaceum $\mathrm{CV} 026$ and the signaling molecule $\mathrm{N}$ hexanoyl-L-homoserine lactone. All the compounds were subjected for ADME predictions by computational method.

KEYWORDS: Sulfonyl hydrazone; hydrazide; MIC; antibacterial activity; quorum sensing.

\section{INTRODUCTION}

The infection created by microorganisms, for example bacteria or fungi, has been regarded as one of the major worldwide medical conditions. Currently, antimicrobial resistance causes 700,000 deaths by the year, and the number is anticipated to rise to 10 million in 2050 [1]. Because of the antibiotic resistance, biofilm formation and increasing number of multi-drug microbial pathogens, scientists have focused on creating novel, less toxic more significant antimicrobial drugs.

Quorum sensing, is a bacterial communication mechanism that regulates gene expression depending on population density and signal molecule secretion [2], also assumed to be responsible for bacterial virulence [3]. In Gram negative bacteria, quorum sensing occurs via acyl homoserine lactone (AHL) signal molecules. While Chromobacterium violaceum (C. violaceum, wild type) is a strain capable of producing short chain AHL and purple colored violacein pigment; mutant $C$. violaceum $\mathrm{CV} 02$ is a biosensor strain that does not produce AHL and pigments but can produce violacein pigment by recognizing short-chain AHL molecules (AHL molecules with chain length C4 to C8) [4]. Quorum sensing inhibition is a powerful route for anti-pathogenic drugs development and control of microbial infections [5].

Ever since the discovery of antibacterial properties of the red dye Prontosil, sulfonamide drugs have been used to treat and prevent bacterial infections in humans [6,7]. Sulfonamides and related sulfonyl derivatives are established in a number of active pharmacophores and shows wide-ranging property (Figure 1). Sulfonyl hydrazones derived from sulfonamides have similar chemical and biological properties and exhibited antidepressant [8], antioxidant [9], anticancer [10] anti-carbonic anhydrase [11,12] and especially antibacterial activities $[13,14]$.

In continuation of our researchs on bioactive molecules, we designed the synthesis with a series of sulfonyl hydrazone derivatives with the antibacterial activity evaluation. Many antibacterial activity studies indicate the electron-withdrawing groups on hydrazone compounds enhance antibacterial activity [15-17].

How to cite this article: Şenkardeş S, Kıymacı ME, Kale K, Kozanoğlu IM, Kaşkatepe B, Küçükgüzel ŞG. Synthesis, structural elucidation and biological activities of some novel sulfonyl hydrazones as antibacterial agents. J Res Pharm. 2021; 25(2): $135-141$. 
The presence of fluorine and nitro groups in the targeted compounds has been chosen due to activityenhancing properties. We obtained a series of original sulfonyl hydrazones (3a-h) and characterized their structures by spectroscopic techniques. We investigated their antibacterial activities against Escherichia coli ATCC 25922, Staphylococcus aureus ATCC 29213, methicillin-resistant Staphylococcus aureus ATCC 43300, Pseudomonas aeruginosa ATCC 27853, Enterococcus faecalis ATCC 29212 and Klebsiella pneumoniae ATCC 13883, by minimal inhibition concentration (MIC) methods for comparison. Additionaly, anti-quorum sensing activity of the derivatives was determined using the reporter biosensor strain Chromobacterium violaceum CV026 reporter strain and the signaling molecule $N$-hexanoyl-L-homoserine lactone agar diffusion assay.

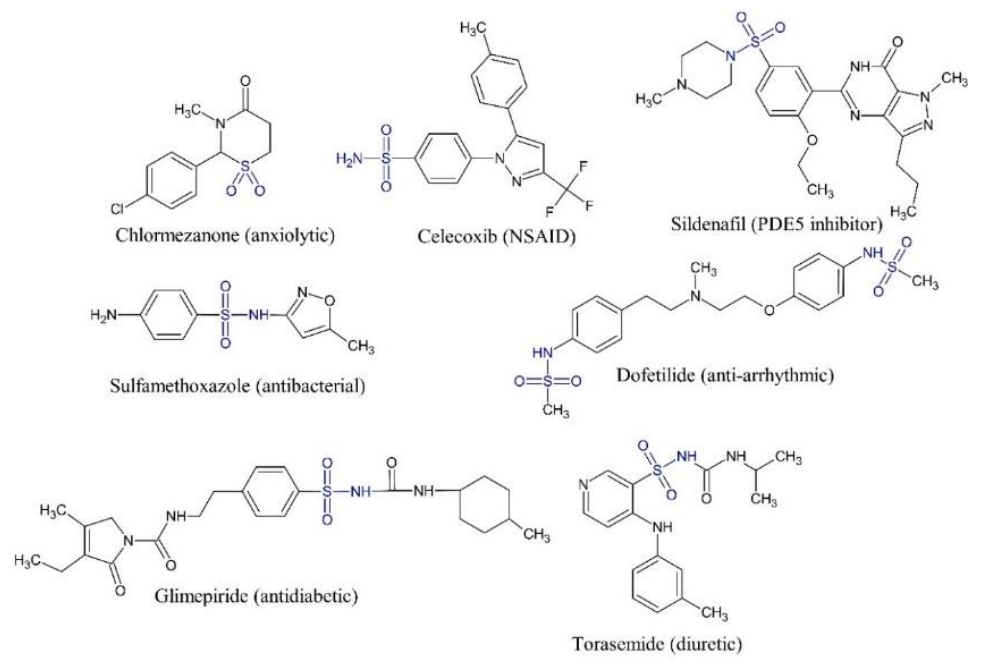

Figure 1. Selected examples of bioactive sulfonyl/sulfonamide derivatives.

\section{RESULTS AND DISCUSSION}

\section{1. Chemistry}

In this present work, new sulfonyl hydrazone derivatives (3a-h) were synthesized for the first time. At first, 4-nitrobenzenesulfonohydrazide (2) was obtained by stirring p-nitrobenzenesulfonyl chloride (1) with hydrazine hydrate in tetrahydrofuran [18]. The reaction of 2 with different aldehydes furnished the corresponding 4-nitro- $N$ '-(substituted arylmethylidene)benzenesulfonohydrazides (3a-h) (Scheme 1) [10,19]. The structures of newly synthesized compounds were supported by spectral data. The FT-IR, ${ }^{1} \mathrm{H}-\mathrm{NMR}$, TOFMS spectra (for $\mathbf{3 b}$ and $\mathbf{3 e}$ ) and elementel analysis results are in aggrement with the proposed structures. In the FT-IR spectra, sharp bands at 3159- $3325 \mathrm{~cm}^{-1}$ due to N-H group and $1602-1626 \mathrm{~cm}^{-1}$ due to $\mathrm{C}=\mathrm{N}$ group are characteristic for sulfonyl hydrazone moiety [10]. In the ${ }^{1} \mathrm{H}-\mathrm{NMR}$ spectra, the signals due to $\mathrm{N}-\mathrm{H}$ and $\mathrm{CH}=\mathrm{N}$ protons are common in all of the compounds appeared as a singlet at $\delta=11.89-12.25 \mathrm{ppm}$ and $\delta=7.92-8.12$ $\mathrm{ppm}$, respectively. The mass spectrum of the compounds $\mathbf{3 b}$ and $\mathbf{3 e}$ chosen as prototypes showed protonated molecular ion peaks $\left([\mathrm{M}+\mathrm{H}]^{+}\right)$in agreement with their molecular formulae.

\section{2. Biological evaluation}

\subsubsection{Antimicrobial activity}

The antimicrobial activity of bacterial species against the compounds are given in Table 1. All of the compounds demonstrated acceptable activity against all microorganisms tested with MIC values varying between 6.25 and $25 \mu \mathrm{g} / \mathrm{mL}$. The best inhibitory effects of all compounds were observed against Pseudomonas aeruginosa having the MIC $6.25 \mu \mathrm{g} / \mathrm{ml}$. According to the results, we may conclude that compounds $3 \mathrm{a}-\mathrm{h}$ have noticeable antibacterial activities.

Additionally, the literature surveys indicated that, sulfonamides have wide antimicrobial activity against Gram-positive and certain Gram-negative bacteria, such as Escherichia coli, Klebsiella, Salmonella and Enterobacter species but show no inhibitory activity against Pseudomonas aeruginosa [20]. This study observed discovery of the more active compounds against Pseudomonas aeruginosa though structural modifications and derivatization. 


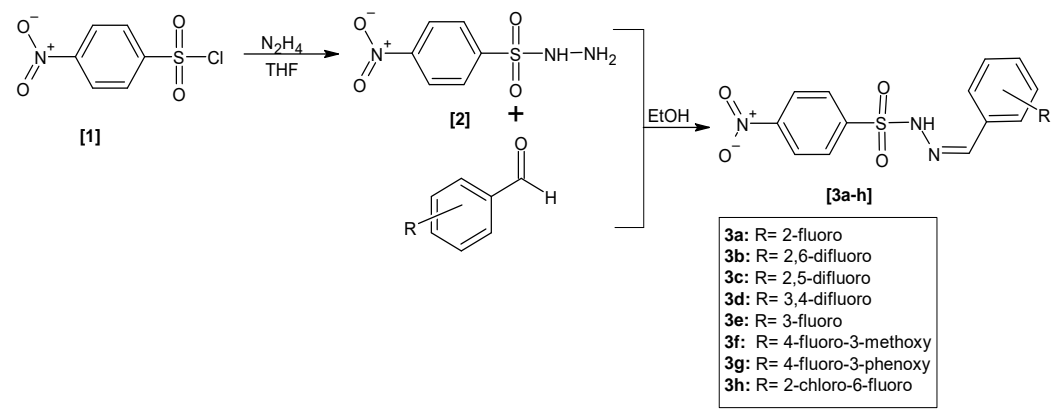

Scheme 1. Synthesis of title compounds (3a-h).

Table 1. Antibacterial activities of the compounds as MIC values $(\mu \mathrm{g} / \mathrm{ml})$.

\begin{tabular}{ccccccc}
\hline \multirow{2}{*}{ Compounds } & \multicolumn{7}{c}{ Microorganisms } \\
\cline { 2 - 7 } & E.c. & S.a. & S.a. ${ }^{*}$ & P.a. & E.f. & K.p. \\
\hline 3a & 25 & 12.5 & 12.5 & 12.5 & 12.5 & 12.5 \\
3b & 25 & 12.5 & 12.5 & 6.25 & 12.5 & 12.5 \\
3c & 12.5 & 12.5 & 12.5 & 6.25 & 12.5 & 12.5 \\
3d & 12.5 & 12.5 & 12.5 & 6.25 & 12.5 & 12.5 \\
3e & 12.5 & 12.5 & 12.5 & 6.25 & 12.5 & 12.5 \\
3f & 12.5 & 12.5 & 12.5 & 6.25 & 12.5 & 12.5 \\
3g & 25 & 12.5 & 12.5 & 6.25 & 12.5 & 12.5 \\
3h & 25 & 12.5 & 12.5 & 6.25 & 12.5 & 25 \\
\hline E.c., Escherichia coli ATCC 25922; S.a., Staphylococcus aureus ATCC29213; S.a. *, Methicillin- \\
resistant Staphylococcus aureus ATCC 43300; P.a., Pseudomonas aeruginosa ATCC 27853; E.f., \\
Enterococcus faecalis ATCC 29212; K.p., Klebsiella pneumoniae ATCC 13883
\end{tabular}

\subsubsection{Anti-quorum sensing activity}

In this study, anti-quorum sensing activity of the test materials at highest and subMIC concentrations was evaluted. It was determined that compounds at subMIC concentrations did not effective activity on quorum sensing mechanism signal molecule $N$-hexanoyl-L-homoserine lactone but the stock concentrations $(400 \mu \mathrm{g} / \mathrm{mL})$ of the compounds showed quorum sensing inhibitory activity. This effect has appeared in the form of inhibition of violacein pigment formation without stopping the growth of bacteria although $N$ hexanoyl-L-homoserine lactone signal molecule and C. violaceum CV026 were together in the environment (Figure 2). The best activity result was obtained from compound $\mathbf{3 g}$ with 4-fluoro-3-phenoxyphenyl substitutent. Other substances were found to have anti-quorum sensing activity at $400 \mu \mathrm{g} / \mathrm{mL}$ concentration.

\subsection{ADME properties}

Lately, research groups have used the potential of chemoinformatics tools to develop a rational drug design method. Currently, these tools have been used in conjunction with experimental testing. Therefore, in silico pharmacokinetic properties of all the novel compounds were evaluated using the Osiris Property Explorer (http://www.openmolecules.org/datawarrior/) and Swiss ADME programs http:/ / www.swissadme.ch/) (Table 2) [21].

According to our prediction result, all of the compounds presented a non-toxic risk profile regarding mutagenic, tumourigenic, irritant and reproductive effects. Also, none of the compounds violated rule of five, that proposed for an acceptable theoretical bioavailability [22]. The predicted $\log P$ values of the compounds were in the range of 1.38-2.06, less than 5. The calculated percent absorption (\% ABS) of all derivatives ranged between $66.92 \%$ and $70.11 \%$, indicating that these compounds have good permeability in the cellular membrane. The topological polar surface area (TPSA) is known as a good mark of absorption in the intestine (less than $140 \AA^{2}$ ) and blood-brain barrier penetration $\left(\leq 60 \AA^{2}\right)$. ABS\% was calculated according to the corresponding literature method [23]. According to predicted data, these compounds have good intestinal absorption and do not have sufficient blood-brain barrier penetration. 


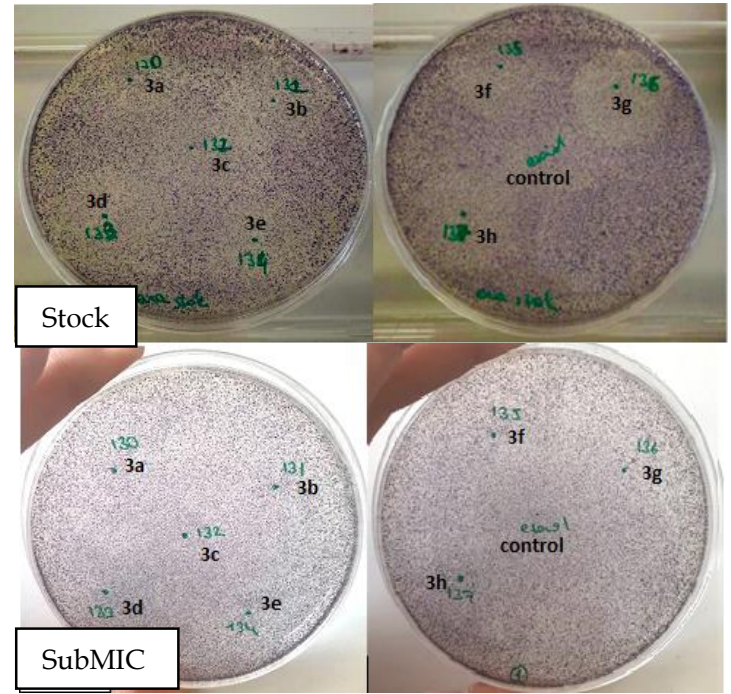

Figure 2. Anti-quorum sensing activity of test materials on violacein pigment production.

Table 2. Lipinski's rule and pharmacokinetic parameters for compounds $\mathbf{3 a - h}$.

\begin{tabular}{|c|c|c|c|c|c|c|c|c|c|c|c|c|c|}
\hline \multirow[b]{2}{*}{ Comp. } & \multicolumn{4}{|c|}{ Toxicity Risks ${ }^{a}$} & \multicolumn{9}{|c|}{ ADME properties ${ }^{b}$} \\
\hline & $\mathbf{M}$ & $\mathbf{T}$ & IR & RE & $\begin{array}{l}\text { MW } \\
\leq 500\end{array}$ & $\begin{array}{c}\operatorname{cLog} P a \\
<5\end{array}$ & $\begin{array}{c}\log S^{a} \\
>-4\end{array}$ & $\begin{array}{l}\mathrm{RB}^{\mathrm{b}} \\
\leq 10\end{array}$ & $\begin{array}{c}\mathrm{HD}^{\mathrm{b}} \\
\leq 5\end{array}$ & $\begin{array}{l}\mathbf{H A}^{\mathrm{b}} \\
\leq 10\end{array}$ & $\begin{array}{c}M^{b} \\
40-130\end{array}$ & $\% \mathrm{Abs}$ & $\begin{array}{l}\text { TPSA }^{b} \\
\leq 140 \AA^{2}\end{array}$ \\
\hline $3 a$ & & & & & 323 & 1.45 & -2.69 & 5 & 1 & 6 & 79.29 & 70.11 & 112.73 \\
\hline $3 b$ & & & & & 341 & 1.55 & -3.00 & 5 & 1 & 7 & 79.25 & 70.11 & 112.73 \\
\hline $3 c$ & & & & & 341 & 1.55 & -3.00 & 5 & 1 & 7 & 79.25 & 70.11 & 112.73 \\
\hline $3 d$ & & & & & 341 & 1.55 & -3.00 & 5 & 1 & 7 & 79.25 & 70.11 & 112.73 \\
\hline $3 e$ & & & & & 323 & 1.45 & -2.69 & 5 & 1 & 6 & 79.79 & 70.11 & 112.73 \\
\hline $3 f$ & & & & & 353 & 1.38 & -2.70 & 6 & 1 & 7 & 85.78 & 66.92 & 121.96 \\
\hline $3 g$ & & & & & 357 & 2.06 & -3.42 & 7 & 1 & 7 & 105.81 & 66.92 & 121.96 \\
\hline $3 h$ & & & & & 357 & 2.06 & -3.42 & 5 & 1 & 6 & 84.30 & 70.11 & 112.73 \\
\hline
\end{tabular}

: not toxic ; M: mutagenic; T: tumorigenic; IR: irritant; RE: reproductive effective; MW: Molecular weight; $\operatorname{cLog} P$ : calculated $\log P$; S: Solubility; RB: Number of rotatable bonds; HD: Number of hydrogen donors; HA: Number of hydrogen acceptors; MR: Molar refractivity; TPSA: Topological polar surface area; aParameters calculated using OSIRIS b Parameters calculated using SwissADME

\section{CONCLUSION}

In this study, our results displayed that aromatic sulfonyl hydrazones with a nitro group had remarkable antibacterial activity and were active against drug-resistant pathogens, such as methicillinresistant Staphylococcus aureus (MRSA) and Pseudomonas aeruginosa. Additionaly, the stock concentrations of the compounds showed inhibitory activity on $N$-hexanoyl-L-homoserine lactone QS signal molecule. It is thought that, more extensive study is needed to optimize the effectiveness of this series of molecules.

\section{MATERIALS AND METHODS}

\subsection{Synthesis}

All chemicals used in this work were purchased from Sigma Aldrich (St. Louis, MO, USA) or Merck (Darmstadt, Germany). Melting points were estimated with a SMP II melting point apparatus and uncorrected. ${ }^{1} \mathrm{H}-\mathrm{NMR}$ spectra were taken on Bruker $300 \mathrm{MHz}$ Ultrashield and Bruker Avance III HD $600 \mathrm{MHz}$ spectrometers. Infrared (IR) spectra were performed using a Shimadzu FTIR 8400 s spectrometer. Elemental analysis was performed using a LECO CHNS-932 analysis system. Mass spectra were recorded on a Xevo G2XS QTof mass spectrometer. Liquid chromatographic system consists of an Agilent Technologies 1100 series and data acquisition was done with the Agilent Chemstation Plus software. Chromatographic separation was performed using a reverse phase Zorbax C8 $(4.0 \times 250 \mathrm{~mm})$ column. The wavelength reading was $254 \mathrm{~nm}$ and the working flow rate was $1 \mathrm{ml} / \mathrm{min}$. All experiments were performed in gradient mode using acetonitrile and water $\left(\mathrm{ACN} / \mathrm{H}_{2} \mathrm{O}\right.$ system was used as gradient system: 0-3 $\min \left(50: 50 \mathrm{ACN} / \mathrm{H}_{2} \mathrm{O}\right) ; 3-6 \min \left(75: 25 \mathrm{ACN} / \mathrm{H}_{2} \mathrm{O}\right)$; 6-12 $\min \left(100: 0 \mathrm{ACN} / \mathrm{H}_{2} \mathrm{O}\right)$. 


\subsubsection{General procedure for the synthesis of 4-nitrobenzenesulfonohydrazide (2)}

The 4-nitrobenzenesulfonohydrazide was produced in good yields ( $85 \%)$ by adding the corresponding p-nitrobenzenesulfonyl chloride in tetrahydrofuran to a slightly excess of hydrazine hydrate solution ( $80 \%)$.

\subsubsection{General procedure for the synthesis $N^{\prime}$-(substituted arylmethylidene)-4-nitrobenzenesulfono hydrazides (3a-h)}

The compounds were synthesized as shown in Scheme 1. A mixture of $p$-nitrobenzenesulfonohydrazide (2) $(0.001 \mathrm{~mol})$ and substituted aldehydes $(0.001 \mathrm{~mol})$ in ethanol was refluxed for 3-4 hours. After completion of reaction, the solvent was evaporated and the solid that formed was filtered, dried and recrystallized from ethanol.

N'-(2-Fluorobenzylidene)-4-nitrobenzenesulfonohydrazide (3a)

Off-white solid; Yield 73\% ; m.p. 144-145 ${ }^{\circ} \mathrm{C}$, Rt (min.): 4.79; FT-IR $\mathrm{U}_{\max }\left(\mathrm{cm}^{-1}\right): 3585\left(\mathrm{OH}, \mathrm{H}_{2} \mathrm{O}\right), 3325$ $(\mathrm{NH}), 1606(\mathrm{C}=\mathrm{N}), 1537,1352\left(\mathrm{NO}_{2}\right), 1329,1165\left(\mathrm{SO}_{2}\right) ;{ }^{1} \mathrm{H}$ NMR $(300 \mathrm{MHz}),\left(\mathrm{DMSO}-d_{6} / \mathrm{TMS}\right) \delta$ ppm: 7.22-7.29 $(2 \mathrm{H}, \mathrm{m}), 7.43-7.51(1 \mathrm{H}, \mathrm{m}), 7.70-7.76\left(1 \mathrm{H}, \mathrm{td}, J_{1}=6 \mathrm{~Hz}, J_{2}=1.5 \mathrm{~Hz}\right), 8.12-8.17(3 \mathrm{H}, \mathrm{m}), 8.41-8.46(2 \mathrm{H}, \mathrm{m}), 12.08(1 \mathrm{H}$, s, NH). Anal. Calcd for $\mathrm{C}_{13} \mathrm{H}_{10} \mathrm{FN}_{3} \mathrm{O}_{4} \mathrm{~S} . \mathrm{H}_{2} \mathrm{O}: \mathrm{C}, 45.75 ; \mathrm{H}, 3.54 ; \mathrm{N}, 12.32 ; \mathrm{S}, 9.39$ Found: $\mathrm{C}, 45.45 ; \mathrm{H}, 3.68 ; \mathrm{N}, 11.88$; S, 9.73 .

\section{N'-(2,6-Difluorobenzylidene)-4-nitrobenzenesulfonohydrazide (3b)}

Yellow solid; Yield 78\% ; m.p. 160-162 ${ }^{\circ} \mathrm{C}$, Rt (min.): 4.73; FT-IR v max $\left(\mathrm{cm}^{-1}\right): 3190$ (NH), 1626 (C=N), 1519,

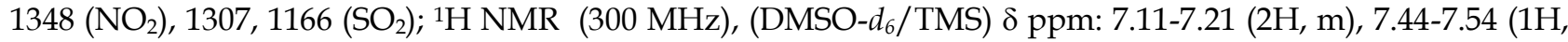
$\mathrm{m}), 8.02(1 \mathrm{H}, \mathrm{s}), 8.10 \quad(2 \mathrm{H}, \mathrm{d}, J=9 \mathrm{~Hz}), 8.44 \quad(2 \mathrm{H}, \mathrm{d}, J=9 \mathrm{~Hz}), 12.16(1 \mathrm{H}, \mathrm{s}, \mathrm{NH})$. Anal. Calcd for $\mathrm{C}_{13} \mathrm{H}_{9} \mathrm{~F}_{2} \mathrm{~N}_{3} \mathrm{O}_{4} \mathrm{~S} .1 / 2 \mathrm{C}_{2} \mathrm{H}_{5} \mathrm{OH}$ : C, 46.15; H, 3.32; N, 11.53; S, 8.80 Found: C, 46.24; H, 2.98; N, 11.29; S, 8.65. TOF/MS: $\mathrm{m} / \mathrm{z}$ for $\mathrm{C}_{13} \mathrm{H}_{9} \mathrm{~F}_{2} \mathrm{~N}_{3} \mathrm{O}_{4} \mathrm{~S}$ [M+H] $]^{+}$: Calc. 342.0354, Found 342.0344.

$N^{\prime}$-(2,5-Difluorobenzylidene)-4-nitrobenzenesulfonohydrazide (3c)

Light yellow solid; Yield 86\% ; m.p. 150-151 ${ }^{\circ} \mathrm{C}$, Rt (min.): 4.74; FT-IR $v_{\max }\left(\mathrm{cm}^{-1}\right): 3159$ (NH), $1604(\mathrm{C}=\mathrm{N})$, 1529, $1346\left(\mathrm{NO}_{2}\right), 1327,1163\left(\mathrm{SO}_{2}\right) ;{ }^{1} \mathrm{H}$ NMR (300 MHz), (DMSO-d $\left.d_{6} / \mathrm{TMS}\right) \delta \mathrm{ppm}$ : 7.11-7.21 (2H, m), 7.44-7.54 $(1 \mathrm{H}, \mathrm{m}), 8.07(1 \mathrm{H}, \mathrm{s}), 8.16(2 \mathrm{H}, \mathrm{d}, J=9 \mathrm{~Hz}), 8.43 \quad(2 \mathrm{H}, \mathrm{d}, J=9 \mathrm{~Hz}), 12.21(1 \mathrm{H}, \mathrm{s}, \mathrm{NH})$. Anal. Calcd for $\mathrm{C}_{13} \mathrm{H}_{9} \mathrm{~F}_{2} \mathrm{~N}_{3} \mathrm{O}_{4} \mathrm{~S} .2 / 3 \mathrm{H}_{2} \mathrm{O}: \mathrm{C}, 44.19 ; \mathrm{H}, 2.95 ; \mathrm{N}, 11.89 ; \mathrm{S}, 9.08$ Found: $\mathrm{C}, 44.23 ; \mathrm{H}, 3.27 ; \mathrm{N}, 11.19 ; \mathrm{S}, 9.27$.

$N^{\prime}$-(3,4-Difluorobenzylidene)-4-nitrobenzenesulfonohydrazide (3d)

Light yellow solid; Yield 88\% ; m.p. 174-176 ${ }^{\circ} \mathrm{C}$, Rt (min.): 4.70; FT-IR $\mathrm{U}_{\max }\left(\mathrm{cm}^{-1}\right): 3171(\mathrm{NH}), 1606(\mathrm{C}=\mathrm{N})$, 1527, $1348\left(\mathrm{NO}_{2}\right), 1305,1163\left(\mathrm{SO}_{2}\right) ;{ }^{1} \mathrm{H}$ NMR (300 MHz), (DMSO-d $d_{6}$ TMS) $\delta$ ppm: 7.45-7.62 (2H, m), 7.62-7.68 $(1 \mathrm{H}, \mathrm{m}), 7.94(1 \mathrm{H}, \mathrm{s}), 8.16(2 \mathrm{H}, \mathrm{d}, \mathrm{J}=9 \mathrm{~Hz}), 8.44(2 \mathrm{H}, \mathrm{d}, \mathrm{J}=9 \mathrm{~Hz}), 12.03(1 \mathrm{H}, \mathrm{s}, \mathrm{NH})$. Anal. Calcd for $\mathrm{C}_{13} \mathrm{H}_{9} \mathrm{~F}_{2} \mathrm{~N}_{3} \mathrm{O}_{4} \mathrm{~S}$ : C, 45.75; H, 266; N, 12.31; S, 9.40 Found: C, 45.98; H, 2.77; N, 12.26; S, 9.66.

N'-(3-Fluorobenzylidene)-4-nitrobenzenesulfonohydrazide (3e)

Off-white solid; Yield 87\% ; m.p. 147-148 ${ }^{\circ} \mathrm{C}$, Rt (min.): 4.80; FT-IR $v_{\max }\left(\mathrm{cm}^{-1}\right): 3578\left(\mathrm{OH}, \mathrm{H}_{2} \mathrm{O}\right), 3190$ $(\mathrm{NH}), 1606(\mathrm{C}=\mathrm{N}), 1537,1352\left(\mathrm{NO}_{2}\right), 1311,1165\left(\mathrm{SO}_{2}\right) ;{ }^{1} \mathrm{H}$ NMR $(300 \mathrm{MHz}),\left(\mathrm{DMSO}-d_{6} / \mathrm{TMS}\right) \delta$ ppm: 7.26-7.38 $(1 \mathrm{H}, \mathrm{m}), 7.39-7.49(3 \mathrm{H}, \mathrm{m}), 7.97(1 \mathrm{H}, \mathrm{s}), 8.15(2 \mathrm{H}, \mathrm{d}, J=9 \mathrm{~Hz}), 8.44(2 \mathrm{H}, \mathrm{d}, J=9 \mathrm{~Hz}), 12.03(1 \mathrm{H}, \mathrm{s}, \mathrm{NH})$. Anal. Calcd for $\mathrm{C}_{13} \mathrm{H}_{10} \mathrm{FN}_{3} \mathrm{O}_{4} \mathrm{~S} . \mathrm{H}_{2} \mathrm{O}: \mathrm{C}, 45.75 ; \mathrm{H}, 3.54 ; \mathrm{N}, 12.31$; , 9.39 Found: $\mathrm{C}, 45.67 ; \mathrm{H}, 3.51 ; \mathrm{N}, 12.11 ; \mathrm{S}, 9.14$. TOF/MS: $\mathrm{m} / \mathrm{z}$ for $\mathrm{C}_{13} \mathrm{H}_{9} \mathrm{~F}_{2} \mathrm{~N}_{3} \mathrm{O}_{4} \mathrm{~S}[\mathrm{M}+\mathrm{H}]^{+}$: Calc. 324.0448, Found 324.0425.

N'-(4-Fluoro-3-methoxybenzylidene)-4-nitrobenzenesulfonohydrazide (3f)

Yellow solid; Yield 74\% ; m.p. 165-167 ㄷ, Rt (min.): 5.40; FT-IR v $\max \left(\mathrm{cm}^{-1}\right)$ : 3200 (NH), $1602(\mathrm{C}=\mathrm{N}), 1518$, $1357\left(\mathrm{NO}_{2}\right), 1346,1165\left(\mathrm{SO}_{2}\right) ;{ }^{1} \mathrm{H}$ NMR $(300 \mathrm{MHz}),\left(\mathrm{DMSO}-d_{6} / \mathrm{TMS}\right) \delta \mathrm{ppm:} 3.86\left(3 \mathrm{H}, \mathrm{s}, \mathrm{CH}_{3}\right), 7.18-7.36(3 \mathrm{H}$, $\mathrm{m}), 7.93(1 \mathrm{H}, \mathrm{s}), 8.14(2 \mathrm{H}, \mathrm{d}, J=9 \mathrm{~Hz}), 8.43(2 \mathrm{H}, \mathrm{d}, J=9 \mathrm{~Hz}), 11.89(1 \mathrm{H}, \mathrm{s}, \mathrm{NH})$. Anal. Calcd for $\mathrm{C}_{14} \mathrm{H}_{12} \mathrm{FN}_{3} \mathrm{O}_{5} \mathrm{~S}: \mathrm{C}$, 47.59; H, 3.42; N, 11.89; S, 9.08 Found: C, 48.07; H, 3.56; N, 11.71; S, 9.00.

$N^{\prime}$-(4-Fluoro-3-phenoxybenzylidene)-4-nitrobenzenesulfonohydrazide (3g)

Off-white solid; Yield 85\% ; m.p. 147-148 ${ }^{\circ} \mathrm{C}$, Rt (min.): 5.42; FT-IR $v_{\max }\left(\mathrm{cm}^{-1}\right): 3182(\mathrm{NH}), 1608(\mathrm{C}=\mathrm{N})$, 1535, $1336\left(\mathrm{NO}_{2}\right), 1303,1166$ (SO $)$; ${ }^{1} \mathrm{H}$ NMR (600 MHz), (DMSO-d $/$ TMS) $\delta$ ppm: 7.01 (2H, d, J=9 Hz), 7.19 $(2 \mathrm{H}, \mathrm{t}, J=8.4 \mathrm{~Hz}), 7.40-7.47(4 \mathrm{H}, \mathrm{m}), 7.92(1 \mathrm{H}, \mathrm{s}), 8.06(2 \mathrm{H}, \mathrm{d}, \mathrm{J}=9 \mathrm{~Hz}), 8.39(2 \mathrm{H}, \mathrm{d}, J=9 \mathrm{~Hz}), 11.94(1 \mathrm{H}, \mathrm{s}, \mathrm{NH})$. Anal. Calcd for $\mathrm{C}_{19} \mathrm{H}_{14} \mathrm{FN}_{3} \mathrm{O}_{5} \mathrm{~S}$ : C, 54.94; H, 3.40; N, 10.12; S, 7.72 Found: C, 55.30; H, 3.53; N, 9.97; S, 7.72. 
$N^{\prime}$-(2-Chloro-6-fluorobenzylidene)-4-nitrobenzenesulfonohydrazide (3h)

Dark green solid; Yield 89\% ; m.p. 186-188 ${ }^{\circ} \mathrm{C}$, Rt (min.): 4.87; FT-IR $v_{\max }\left(\mathrm{cm}^{-1}\right): 3176(\mathrm{NH}), 1604(\mathrm{C}=\mathrm{N})$, 1519, $1355\left(\mathrm{NO}_{2}\right), 1307,1170\left(\mathrm{SO}_{2}\right) ;{ }^{1} \mathrm{H}$ NMR $(600 \mathrm{MHz}),\left(\mathrm{DMSO}-d_{6} / \mathrm{TMS}\right) \delta$ ppm: $7.26(1 \mathrm{H}, \mathrm{t}, J=9 \mathrm{~Hz}), 7.19$ $(1 \mathrm{H}, \mathrm{d}, J=7.8 \mathrm{~Hz}), 7.44-7.47(1 \mathrm{H}, \mathrm{m}), 8.11(2 \mathrm{H}, \mathrm{d}, J=9 \mathrm{~Hz}), 8.12(1 \mathrm{H}, \mathrm{s}), 8.45(2 \mathrm{H}, \mathrm{d}, J=9 \mathrm{~Hz}), 12.25(1 \mathrm{H}, \mathrm{s}, \mathrm{NH})$. Anal. Calcd for $\mathrm{C}_{19} \mathrm{H}_{14} \mathrm{FN}_{3} \mathrm{O}_{5} \mathrm{~S} .1 / 3 \mathrm{C}_{2} \mathrm{H}_{5} \mathrm{OH}: \mathrm{C}, 54.94 ; \mathrm{H}, 3.40 ; \mathrm{N}, 10.12 ; \mathrm{S}, 7.72$ Found: $\mathrm{C}, 44.36 ; \mathrm{H}, 2.77 ; \mathrm{N}, 9.97$; S, 7.72 .

\subsection{Biological studies}

\subsubsection{Antibacterial activity test}

The synthesized compounds were tested for in vitro antibacterial activity against Escherichia coli ATCC 25922, Staphylococcus aureus ATCC 29213, methicillin-resistant Staphylococcus aureus ATCC 43300, Pseudomonas aeruginosa ATCC 27853, Enterococcus faecalis ATCC 29212 and Klebsiella pneumoniae ATCC 13883 with using micro broth dilution method according to the methodology described in the literature [24]. The minimum concentration that inhibits bacterial growth accepted as minimal inhibition concentration (MIC). The results were evaluated according to the EUCAST clinical control tables [25].

\subsubsection{Anti-quorum sensing activity determination}

$\mathrm{N}$-Hexanoyl-L-homoserine lactone was used as signal molecule for biosensor strain. The fresh culture of C. violaceum CV026 at $30^{\circ} \mathrm{C}$ for 18 hours was taken, and adjusted to Mc Farland 0.5 density $\left(10^{8} \mathrm{cfu} / \mathrm{mL}\right)$. $100 \mu \mathrm{L}$ C. violaceum CV026, and $50 \mu \mathrm{L} N$-hexanoyl-L-homoserine lactone was added to $10 \mathrm{~mL}$ soft Luria Bertani agar $(0.9 \%)$ medium and poured into petri plates after vortexing thoroughly. The test materials at highest concentrations (stock) and subMIC concentrations was dropped on $(20 \mu \mathrm{L})$ agar plates and incubated $30^{\circ} \mathrm{C}$ for 48 hours. The test was carried out three times and ethanol was used as control [26].

Acknowledgements: This research work was supported by TÜBİTAK 2209-A University Students Research Projects Support Program. (Project application no: 1919B012000350).

Author contributions: Concept-S.Ş., Ş.G.K, Design-S.Ş., Writing-S.Ş., B.K, M.E.K, Ş.G.K., Resources- S.Ş.- K.K.-M.İ.K, Literature Search-S.Ş., B.K, M.E.K, K.K., İ.M.K. Analysis and/or Interpretation-S.Ş., B.K, M.E.K, K.K., İ.M.K., Critical Reviews-S.Ş., B.K, M.E.K, Ş.G.K- İ.M.K, K.K.

Conflict of interest statement: The authors declared no conflict of interest.

\section{REFERENCES}

[1] Tagliabue A, Rappuoli R. Changing priorities in vaccinology: Antibiotic resistance moving to the top. Front Immunol. 2018; 9: 1068. [CrossRef]

[2] Papenfort K, Bassler BL. Quorum sensing signal-response systems in Gram-negative bacteria. Nat Rev Microbiol. 2016; 14(9): 576-588. [CrossRef]

[3] Sakuragi Y, Kolter R. Quorum-sensing regulation of the biofilm matrix genes (pel) of Pseudomonas aeruginosa. J Bacteriol. 2007; 189(14): 5383-5386. [CrossRef]

[4] McClean KH, Winson MK, Fish L, Taylor A, Chhabra SR, Camara M, Daykin M, Lamb JH, Swift S, Bycroft BW, Stewart GS, Williams P. Quorum sensing and Chromobacterium violaceum: Exploitation of violacein production and inhibition for the detection of $\mathrm{N}$-acylhomoserine lactones. Microbiology. 1997; 143(12): 3703-3711. [CrossRef]

[5] Rafiee F, Haghi F, Bikas R, Heidari A, Gholami M, Kozakiewicz A, Zeighami H. Synthesis, characterization and assessment of anti-quorum sensing activity of copper(II)-ciprofloxacin complex against Pseudomonas aeruginosa PAO1. AMB Express. 2020; 10(1): 82. [CrossRef]

[6] Aminov RI. A brief history of the antibiotic era: Lessons learned and challenges for the future. Front Microbiol. 2010; 1: 134. [CrossRef]

[7] Fair RJ, Tor Y. Antibiotics and bacterial resistance in the 21st century. Perspect Medicin Chem. 2014; 6: 25-64. [CrossRef]

[8] De Oliveira KN, Costa P, Santin JR, Mazzambani L, Bürger C, Mora C, Nunes RJ, Souza MM. Synthesis and antidepressant-like activity evaluation of sulphonamides and sulphonyl-hydrazones. Bioorganic Med Chem. 2011; 19(14): 4295-4306. [CrossRef] 
[9] Karaman N, Oruç-Emre EE, Sıcak Y, Çatıkkaş B, Karaküçük-İyidoğan A, Öztürk M. Microwave-assisted synthesis of new sulfonyl hydrazones, screening of biological activities and investigation of structure-activity relationship. Med Chem Res. 2016; 25(8): 1590-1607. [CrossRef]

[10] Şenkardeş S, Han Mİ, Kulabaş N, Abbak M, Çevik Ö, Küçükgüzel İ, Küçükgüzel ŞG. Synthesis, molecular docking and evaluation of novel sulfonyl hydrazones as anticancer agents and COX-2 inhibitors. Mol Divers. 2020; 24(3): 673689. [CrossRef]

[11] Özmen Özdemir Ü, Altuntaş A, Gündüzalp AB, Arslan F, Hamurcu F. New aromatic/heteroaromatic propanesulfonylhydrazone compounds: Synthesis, physical properties and inhibition studies against carbonic anhydrase II (CAII) enzyme. Spectrochim Acta - Part A Mol Biomol Spectrosc. 2014; 128: 452-460. [CrossRef]

[12] Gündüzalp AB, Parlakgümüş G, Uzun D, Özmen ÜÖ, Özbek N, Sarı M, Tunç T. Carbonic anhydrase inhibitors: Synthesis, characterization and inhibition activities of furan sulfonylhydrazones against carbonic anhydrase I (hCA I). J Mol Struct. 2016; 1105: 332-340. [CrossRef]

[13] Aslan HG, Karacan N. Aromatic sulfonyl hydrazides and sulfonyl hydrazones: Antimicrobial activity and physical properties. Med Chem Res. 2013; 22(3): 1330-1338. [CrossRef]

[14] Dodoff NI, Özdemir Ü, Karacan N, Georgieva MC, Konstantinov SM, Stefanova ME. Schiff Bases of Methanesulfonylhydrazine. Synthesis, Spectroscopic Characterization, Conformational Analysis, and Biological Activity. Z Naturforsch B. 1999; 54(12): 1553-1562. [CrossRef]

[15] Muhammad YA, Narang R, Nayak SK, Singh SK. Synthesis, antibacterial activity and molecular docking studies of $N^{\prime}$-benzylidene/ $N^{\prime}$-(1-phenylethylidene)hexa-2,4-dienehydrazide derivatives. J Chem Pharm Res. 2016; 8(3): 930937.

[16] Nastasa C, Tiperciuc B, Duma M, Benedec D, Oniga O. New hydrazones bearing thiazole scaffold: Synthesis, characterization, antimicrobial, and antioxidant investigation. Molecules. 2015; 20(9): 17325-17338. [CrossRef]

[17] Ibrahim HM, Behbehani H, Elnagdi MH. Approaches towards the synthesis of a novel class of 2-amino-5arylazonicotinate, pyridazinone and pyrido[2,3-d]pyrimidine derivatives as potent antimicrobial agents. Chem Cent J. 2013; 7(1): 1-16. [CrossRef]

[18] Fernandes TB, Cunha MR, Sakata RP, Candido TM, Baby AR, Tavares MT, et al. Synthesis, molecular modeling, and evaluation of novel sulfonylhydrazones as acetylcholinesterase inhibitors for Alzheimer's disease. Arch Pharm (Weinheim). 2017; 350(11): 1-16. [CrossRef]

[19] Younus HA, Hameed A, Mahmood A, Khan MS, Saeed M, Batool F, Asair A, Mohamad H, Pelletier J, Sevigny J, Iqbal J, al-Rashida M. Sulfonylhydrazones: Design, synthesis and investigation of ectonucleotidase (ALP\&e5'NT) inhibition activities. Bioorg Chem. 2020; 100: 103827. [CrossRef]

[20] Tacic A, Nikolic V, Nikolic L, Savic I. Antimicrobial sulfonamide drugs. Adv Technol. 2017;6(1):58-71.

[21] Daina A, Michielin O, Zoete V. SwissADME: A free web tool to evaluate pharmacokinetics, drug-likeness and medicinal chemistry friendliness of small molecules. Sci Rep. 2017; 7: 42717. [CrossRef]

[22] Lipinski CA, Lombardo F, Dominy BW, Feeney PJ. Experimental and computational approaches to estimate solubility and permeability in drug discovery and development settings. Adv Drug Deliv Rev. 2012; 64(SUPPL.): 4-17. [CrossRef]

[23] Zhao YH, Abraham MH, Le J, Hersey A, Luscombe CN, Beck G, Sherborne B, Cooper I. Rate-limited steps of human oral absorption and QSAR studies. Pharm Res. 2002; 19(10): 1446-1457. [CrossRef]

[24] ISO 20776-1 (2006). Clinical laboratory testing and in vitro diagnostic test systems - Susceptibility testing of infectious agents and evaluation of performance of antimicrobial susceptibility test devices -Part 1: Reference method for testing the in vitro activity of antimicrobial agents against rapidly growing aerobic bacteria involved in infectious diseases.

[25] The European Committee on Antimicrobial Susceptibility Testing (EUCAST). Routine and extended internal quality control for MIC determination and disk diffusion as recommended by EUCAST. Version 8.0, 2018.

[26] Erdönmez D, Rad AY, Aksöz N. Anti-Quorum sensing potential of antioxidant quercetin and resveratrol. Brazilian Arch Biol Technol. 2018; 61: 18160756. [CrossRef]

This is an open access article which is publicly available on our journal's website under Institutional Repository at http://dspace.marmara.edu.tr. 